\title{
An Integrated Architecture for Learning of Reactive Behaviors based on Dynamic Cell Structures
}

\author{
Jörg Bruske, Ingo Ahrns and Gerald Sommer
}

Institut für Informatik und Praktische Mathematik Christian-Albrechts-Universität Kiel

Preußerstraße 1-9

D-24105 Kiel

Bericht Nr. 9604

Mai 1996

Dieser Bericht ist als persönliche Mitteilung aufzufassen

This work has been submitted to Elsevier Scienc Publishers for possible publication. Copyright may be transferred without notice, after which this version may no longer be accessible. 
This report is replaced by the article

\title{
An Integrated Architecture for Learning of Reactive Behaviors based on Dynamic Cell Structures
}

in Robotics and Autonomous Systems, Elsevier Science B.V., to appear.

All rights concerning reprints of any part and electronic distribution have been granted to Elsevier Science B.V.

C1997 Elsevier Science B.V.

\begin{abstract}
In this contribution we want to draw the readers attention to the advantages of controller architectures based on Dynamic Cell Structures (DCS) [5] for learning reactive behaviors of autonomous robots. These include incremental on-line learning, fast output calculation, a flexible integration of different learning rules and a close connection to fuzzy logic. The latter allows for incorporation of prior knowledge and to interpret learning with a DCS as fuzzy rule generation and adaptation.

After successful applications of DCS to tasks involving supervised learning, feedback error learning and incremental category learning, in this article we take reinforcement learning of reactive collision avoidance for an autonomous mobile robot as a further example to demonstrate the validity of our approach. More specifically, we employ a REINFORCE [23] algorithm in combination with an Adaptive Heuristic Critique (AHC) [21] to learn a continuous valued sensory motor mapping for obstacle avoidance with a TRC Labmate from delayed reinforcement. The sensory input consists of eight unprocessed sonar readings, the controller output is the continuous angular and forward velocity of the Labmate. The controller and the AHC are integrated within a single DCS network, and the resulting avoidance behavior of the robot can be analyzed as a set of fuzzy rules, each rule having an additional certainty value.
\end{abstract}

